\title{
Spiral Flows at the Cardiovascular System as the Experimental Base of New Cardiac-gadgets Design
}

\author{
A.V. Savelyev ${ }^{\text {a }}$, I.V. Stepanyan ${ }^{\text {b }}$ \\ ${ }^{a}$ Faculty of Philosophy of Moscow State University named after M.V. Lomonosov \\ ${ }^{b}$ Institute of Machine Science named after A.A. Blagonravov of the RAS
}

Received: 23 June 2018; Accepted: 16 October 2018; Published: 08 November 2018

\begin{abstract}
The results of studies of the functional mechanodynamics of the arteries of the cardiovascular system are presented. Methods of mathematical neural computer modeling, developed by the authors, were reproduced the features of blood flow in statics and dynamics, taking into account the spiral laying of vessels smooth muscle with the transfer of its relief through a thin endothelial membrane lining the inner surface. It is shown that the the nature of the blood flow significantly differs with the both quantitative and qualitative characteristics from the blood flow without regard to the spiral endothelial relief repeating helical laying of smooth shell. The developed methods of neurocomputer modeling allow reconstruct and detect events to recreate the picture of the functioning of living bio tissue. Based on the obtained results and neural networks modeling, a new paradigm for the design of implantable cardiac-gadgets purposed.
\end{abstract}

Index Terms: Virtual "Revival", cardiac-gadgets, spiral laying of vessels smooth muscle, neural networks modeling, mechanodynamics.

(C) 2018 Published by MECS Publisher. Selection and/or peer review under responsibility of the Research Association of Modern Education and Computer Science.

\section{Introduction}

Known [1] longitudinal spiral nature of the laying of muscle fibers along the large arteries. It is generally accepted that this geometry allows them to more effectively perform the sphincter function [2] or the local microcirculatory pumping function of the bloodstream that relieves the heart muscle [3]. However, in all schemes and drawings in the literature, a smooth surface of the lining of the vessels of the end epithelial tissue

* Corresponding author. Tel.: +62 85250986419

E-mail address: gmkristo@yandex.ru, neurocomp.pro@gmail.com 
lining the vessels is given, even though its elasticity is emphasized (Fig. 1). In connection with these ideas, the designs of existing stents (Fig. 2) do not provide for the adaptation to the spiral nature of the placement of muscle fibers, which are well viewed in Fig. 1.

With continued improvement of information technologies, the urgent task is not only to increase the number of observations of the functioning of a bio-object or its limited area per unit of time, but also to adequately present the information received. The task is complicated by the increase in the number of readings in order to assess the faster components of the change in the state of biotissue, as well as by increasing the resolution of the recorded and modeled information images in each sample. The most complicated object from this point of view is the brain, however, taking into account the specific features of this task for a given object, the methodology for solving it can be quite general to apply to any biotissues inducing cardiac biotissues.

The aim of the work is to investigate the dependence of blood flow in arterial vessels on the structure of the lining inner endothelial membrane in statics and dynamics while reducing the spiral-folded smooth muscle muscles of the vessels. In this regard, to formulate a new paradigm for the design of safe cardiac gadgets of the new generation, implanted in the cardiovascular system.

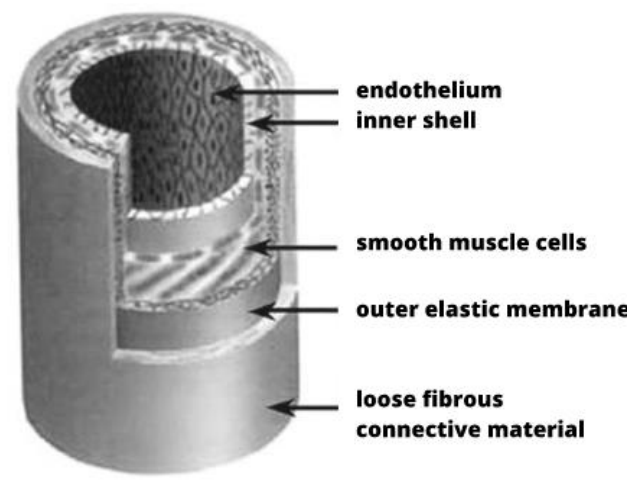

Fig.1. The Structure of the Artery In The Section, Visible Spiral Styling of Smooth Muscle Fibers.

The paper also presents the results of research into the possibilities of alternative ways to solve these problems involving the physiological parameters of simulated biological tissues and the development of a methodology for constructing specialized animation algorithms that reproduce the effects of structural and functional "revitalization" of real-life biological tissue in the nervous and cardiac system. The issues of coordination of functional activity, in this case, electrodynamic, and morph dynamics, are considered.

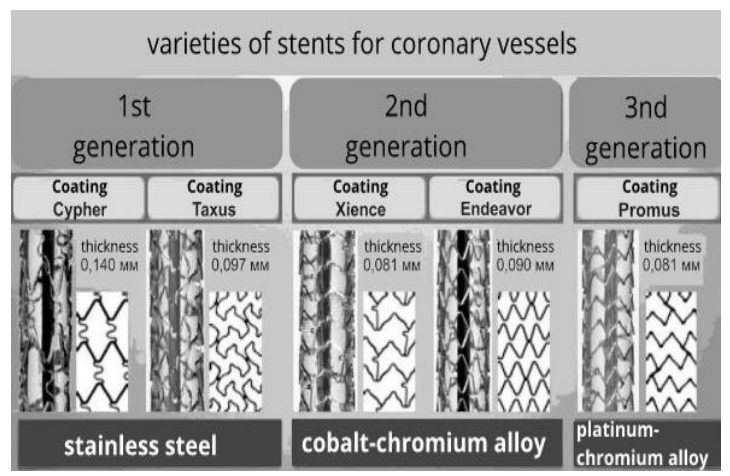

Fig.2. The Variety of Stents of Arterial Vessels used Today With Their Classification by Materials and Configuration. 
The method of cellular neurocomputer collective imitation [4], developed by the authors [5,19,20,30], was used to visualize the mechanical displacements of the spiral muscles during the functioning of the cardiovascular system.

\section{Related Works}

In the article [6] with the technology of manufacturing the fiber optic sensor of pulse waves meeting the requirements of high spatial and temporal resolution, researchers of biomechanical laboratory of Mechanical Engineering Research Institute of the Russian Academy of Sciences (IMASH RAN) outlined ways of mechanopulsography, with the development of the measurement units. Functionally technology can evolve and may be applied in the non-invasive determination of blood pressure no-cuffed method, determining CASP pulse parameters, non-invasive measurement of the concentration of blood sugar and etc [7].

Nakayama $Y$ et al. [8] developed a long in vivo tissue-engineered "Biotube" vascular grafts. In-body tissue architecture (iBTA), a cell-free, in vivo tissue engineering technology that can produce autologous implantable tissues of the desired shape by subcutaneously embedding specially designed molds, was used to develop long tubular collagenous tissues called Biotubes. Spiral molds for long Biotubes were assembled with an outer pipeshaped spiral shell and an inner spiral mandrel, and embedded into subcutaneous pouches of beagle dogs or goats for 1 or 2 months.

At [9] whole-heart spiral simultaneous multi-slice first-pass myocardial perfusion imaging was researched with purpose to develop and evaluate a simultaneous multislice (SMS) spiral perfusion pulse sequence with whole-heart coverage. At result SMS spiral perfusion was produced high-quality perfusion images with wholeheart coverage in a clinical setting with high sampling.

Yahyaie $\mathrm{M}$ et al. at [10] mentioned that most of the research on heart attack prediction has been based on the offline decision-making approach. Experiments on the real data set showed that using the Internet of Things with neural networks predicts a heart attack with acceptable accuracy.

At [11] presents a new deep learning approach for cardiac arrhythmia detection based on long-duration electrocardiography signal analysis. The goal of research was to design a new method based on deep learning to efficiently and quickly classify cardiac arrhythmias. The main contribution was to design Convolutional Neural Network model (1D-CNN). In work [12] was also used a deep learning methods to predict severe complications (mortality, renal failure with a need for renal replacement therapy, and postoperative bleeding leading to operative revision) in post cardio surgical care in a real time. Results were externally retrospectively validated with cases from the published MIMIC-III dataset.

Article [13] mentioned that atrial fibrillation (AF) is one of the most common cardiac arrhythmias. Authors purposed method based on continuous wavelet transform and 2D convolutional neural networks (CNNs) to detect AF episodes on the time-frequency features of the electrocardiogram (ECG). 2D CNN was trained to improve AF detection performance. As the proposed algorithm targets the time-frequency feature of ECG signals rather than isolated atrial or ventricular activity, it has the ability to detect AF episodes for using five beats, suggesting practical applications in medicine.

At work [14] mentioned that knowledge of tissue properties of the abdominal aorta can improve understanding of vascular disease and guide interventional approaches and purposed to assess regional variation in abdominal aortic wall displacement and strain as a function of circumferential position using spiral cine displacement encoding with stimulated echoes. As a result DENSE imaging in the abdominal aortic wall demonstrated that the anterior aortic wall exhibits the greatest displacement, while the lateral wall experiences the largest circumferential strain.

\section{Methods of Reconstruction of The Functioning of a Living Biological Tissue as a Communicative Object}

Shooting a static morphological structure, although in this direction significant success has been achieved 
with the introduction of the methods NMR, ultrasound [15,16] end others, are not in all cases effective diagnosis, especially in the early stages of pathologies. This is explained by the fact that the construction of static even 3D images can show only rather coarse morphological changes characteristic of the deep stages of the development of pathologies. In addition, the reconstruction of images, even the most advanced to date, has a resolution that is far from being able to see the cellular structure of the biological tissue, especially since it is continuously in the dynamics of its current temporal changes. In addition, image restoration algorithms using interpolation and approximation methods are based, as a rule, on optimization procedures that boil down to multiple integrations, which greatly slow down the work of such algorithms and make it very difficult or even impossible to work in real time.

However, the need to use them when playing back a video sequence of multi-dimensional multipoint images greatly complicates such dynamic visualization. It is known that such computational procedures, as a rule, are irregular and, due to large ranges of repeated changes in the input data, reveal high sensitivity to singularities, which causes instability of such procedures, high sensitivity of algorithms to noise and, as a result, low accuracy of the results, reflected in distortion of the obtained images or their interpretations, sometimes to unacceptable degrees.

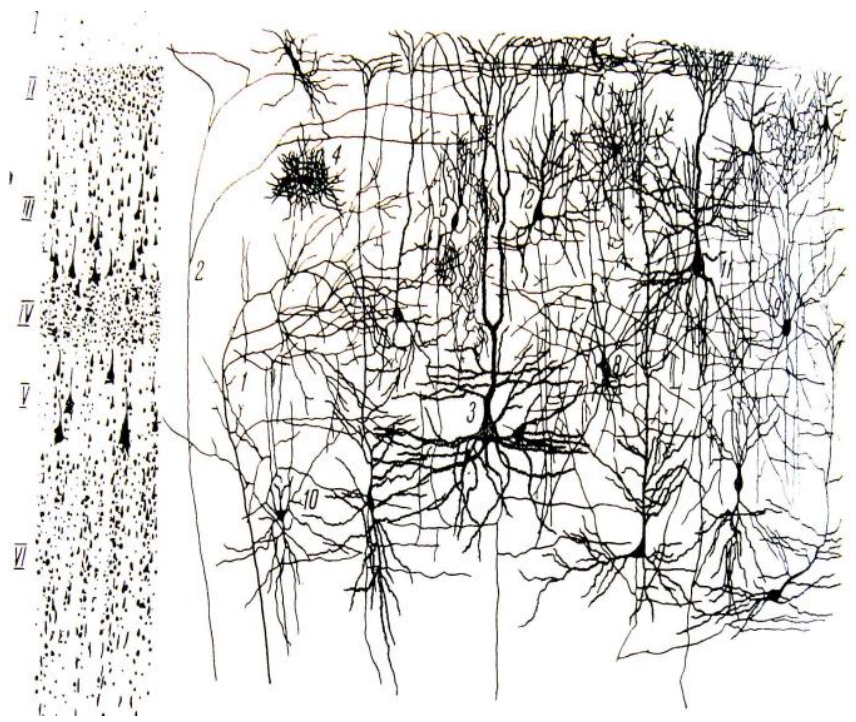

Fig.3. Fragment of the Neural Network of the New Cortex According to G.I. Polyakov. 1 - main and 2 - Associative Afferent Fibers, 3 Pyramidal Neurons of the V layer, 4-10 - Stellate Switching Neurons, 11, 12 - Pyramidal Neurons of the III Layer. On the left are the Cytoarchitectonic Layers I-VI.

Due to the considerable complexity (Fig. 3 - an example of a super complex system) or sometimes the impossibility of observations of this kind on natural objects ("super complex systems", in the terminology of $[17,18]$ ), especially cell dimensions, the development of one of the approaches of which are considered in the presented work. This methodology is conceptual and can be used as a general approach to modeling supercomplex systems in space-time-functional dynamics, as well as an extension of the paradigm of the concept of a neurocomputer [19].

In fig. 4 depicts a preliminary static simulation of a 3D representation of a dendrite of a neuron from microelectronic images of a longitudinal and transverse slice with the dynamic implementation of the procedure for applying an exciting action arising from one point and propagating through it. In fig. 4, for simplicity and more illustrative changes in the distribution of surface potential depending on the configuration of the dendromembrane surface, a fragment of the distal end of the dendrite is presented. 
At the same time, the structure of the dendrite can be even more complex, taking into account possible branches of its central trunk, varying degrees of synaptic occupancy, the degree of development of the spine apparatus, as well as the saturation of the intradendritic neurofilaments and the presence of the microtubule cytoskeleton and microfilamentous filaments, fig. 4.

In addition, metabolic changes of various time scales can be significant, including those comparable to the observation time, in particular, synaptic exocytosis, pinocytosis, internal movements of microcomplexes, neurofilaments and the corresponding mechanical perturbations of the cytoplasm, etc. The developed the principle of superposition allows you to create the effects of "reviving" an individual cell, combining cells [20] to a department or region of the brain.
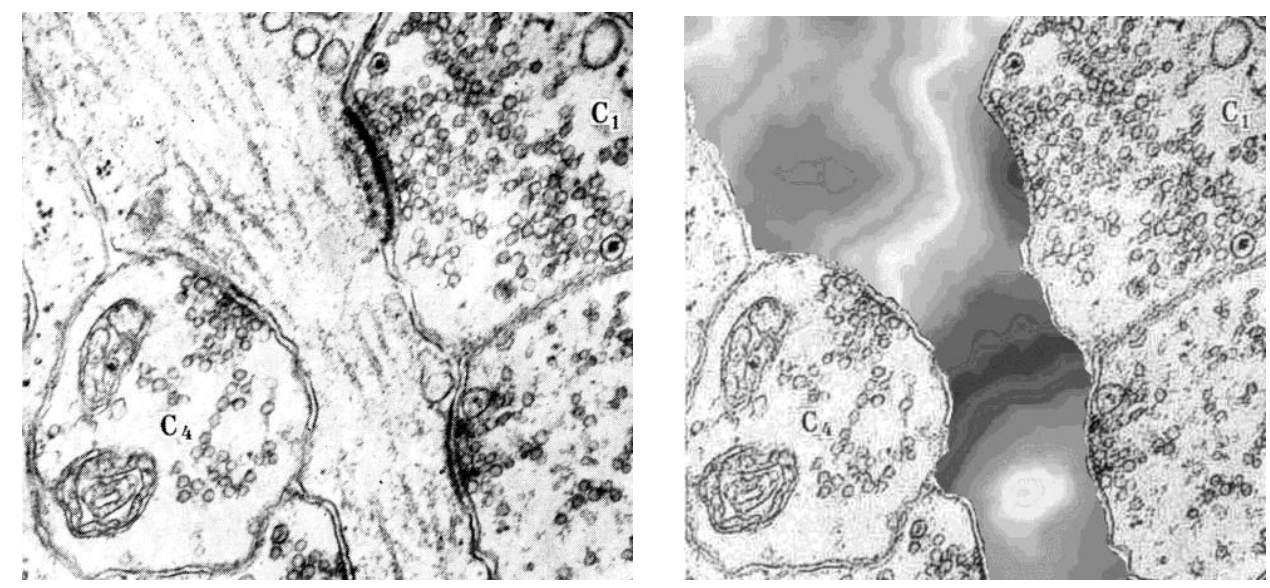

Fig.4. Left - Fragment of the Dendritic Stem, Numerous Micro-Tubules, Microfilament Threads, Inclusions and Heterogeneity of the Cytoplasm are Visible (Microscope Photo of N.S. Koshitsin). Right - According Distribution of Potential on the Dendrite Membrane, Free from Internal Microfilaments.

It can be seen that the excitation extends not only along the dendrite, which is recognized at best by taking into account the propagation along the dendrite in primitive known models, but also in the transverse direction along the dendrite surface relative to its longitudinal axis. And this process takes a certain finite time.

In addition, it can be noted that the propagation along the dendrite occurs faster in the distal direction than in the proximal one, which is apparently due to its narrowing, as well as a change in the nature and density of the perfusing filaments towards the distal end, as noted by a number of researchers [21].

The developed methods of neurocomputer modeling allow not only to reconstruct, but also to generally detect these events and to recreate the picture of the functioning of living biotissue.

The conceptually collective properties of the nervous tissue are displayed in the neuromodel of intersynaptic interactions on one dendrione of the neuron. In this case, the dendrite of the neuron with regard to the microfilament filling is represented as a collective stratified system, functioning due to the communicative exchanges of metabolic, mechanical and electrical interactions.

In the neural model the electrical collective interactions of synapses through the dendrite are represented through mutual influences on the individual parameters and qualitative expression of the post-synaptic potentiation of each synaptic element. 


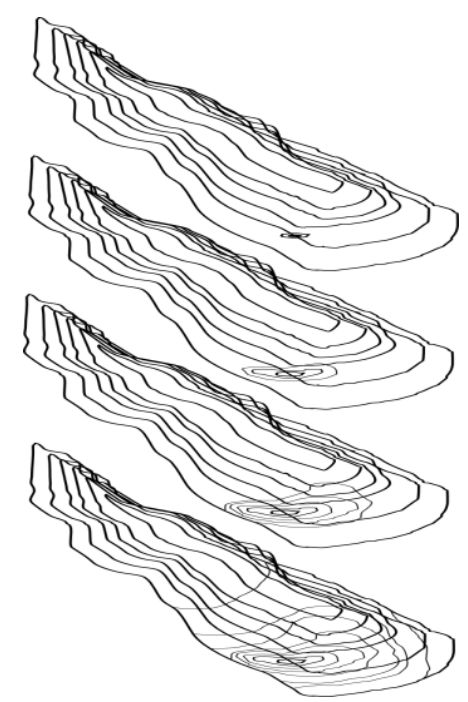

Fig.5. Static 3D Reconstruction of the Neural Dendrite. Thick Profiles Depict the Morphology of Slices of a Dendritic Trunk Parallel Planes along the Dendrite. Thin Profiles Indicate Potential Isolines of Excitation Propagating through it from one Point, View from the External Surface of the Dendromembrane.

In such a neural model, due to its structure, with the help of simple parameter settings, it is possible to take into account the influence of not only the internal microfilaments, but also the external microarchitecture of the dendrite and the whole dendrion of the modeled neuron.

The injection of electricity was modeled in the dotted version. In a more complicated version (Fig. 6), it can be a multipoint design of injected currents from multiple synapses and a distributed injection area for each of the synaptic contacts.

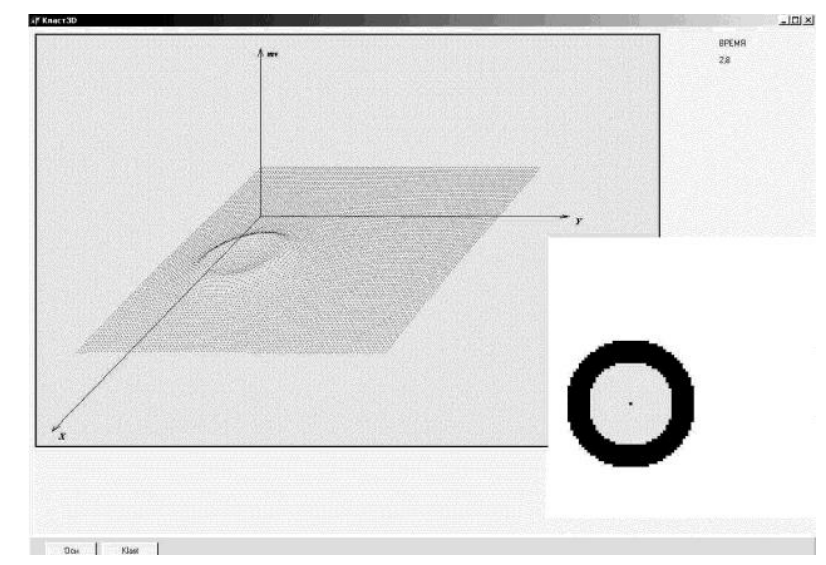

Fig.6. The Most Accurate Reproduction of Exocytosis as the Propagating Waves in a Multipoint Version of Neural Network Modeling.

On Fig. 7, the core region has the form of ridge along which the vesicles of $10 \mathrm{~nm}$ in diameter are located. The crater organization of membrane in Fig. 6 (right) corresponds to the fusion of the vesicles with the presynaptic membrane and postsynaptic disclosure them into space with neurotransmitter release.

Such a distributed injection region can be very well coordinated with the digital representation of images, since, as is well known, injection of current into post-caps is carried out by chemo-dependent membrane ion 
channels activated by mediator molecules [22]. Since the distribution of such channels in the postsynaptic membrane section is discrete and is carried out only by vesicular exocytosis (Fig. 6), this may cause pixel-bypixel matching with the matrix representation of the postsynaptic furniture in its simplest representation with minimal computational resources.
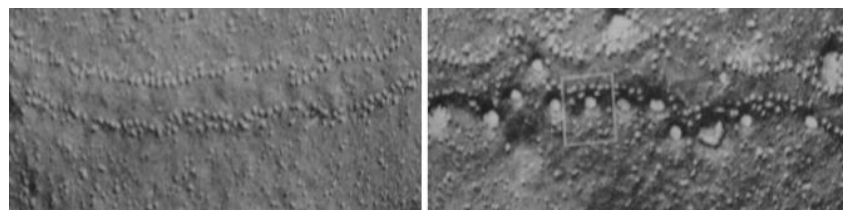

Fig.7. left - Vesicular Exocytosis, View from the synaptic Cleft of the Post-Synaptic Membrane. Electron Micrograph of the Neuromuscular Endplate. (Photo by Heuser J.E., et al. [22]). Right - the same on an Enlarged Scale, the Opening of the Vesicle Attached to the Synaptic Membrane from the Intrasynaptic Space (Circled in Square) is Visible.

A more complex version of the animation method involves so-called "double overlay" when the dynamic of animation superimposed not onto the morphologically static object but parallelly onto the same time slots with reproduced dynamics of its forms. On Fig. 8 the transformation and cell deformation at electrogenesis and antidromic returns to background refractory period is visible.
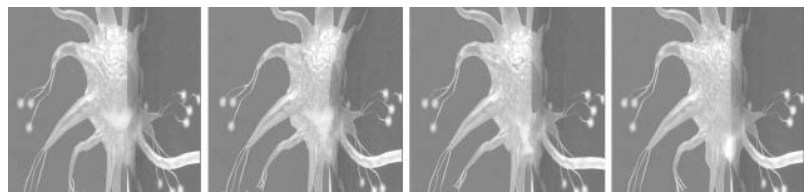

Fig.8. Fragment of Animation of 3D-Reconstruction of Neuron during Axon Discharge

The object morphology is modeled from the set of morphemes of the corresponding database and includes 3D reconstructions of objects based on available data, for example, various electronic micrographs, approximations and interpolations of the video sequence of forms, including, based on heuristic procedures. Form design is performed by a polygonal method or by the finite element method (rigging and rendering [23, 24]).

An example of the implementation of the methodology for coordinating the electrical activity of a neuron as a collective element of individual-synaptic communications with its synaptic-dendritic morphodynamics was used by us in the neuromodel which reproduces the reflection in the electrical activity of the neuron of the morphodynamic processes of slow synaptic migration along the surface of the dendritic processes, correlated with the electrical synaptic activation. The matching is performed by a custom dendrite finite-difference model built on the time shift operators [25] by changing its wave characteristics [26] in the inter-synaptic sections under the influence of complex information processing of the input stream of spike sequences over a set of parallel collectively interacting synaptic channels. The change of parameters, time of propagation of waves of electrical activity over the dendrite surface and qualitative transformation of waves in the process of propagation simulate inter-synaptic collective communication not only in functional, but also in inter-spatial terms, expressed in the dynamics of the inter-synaptic distances of the pattern of mutual arrangement of synapses on the dendrite.

\section{Results}

The developed principles of modeling and complex of algorithms have been successfully applied to solve some problems in cardiodynamics [19,20] and was applied to the therapeutic practice, in particular for the 
diagnosis of the morphological and functional spatial position of the sources of arrhythmia in myocardial tissue which representing as a pool of myocytes or individual myocytes that was lost the ability of the electric and/or mechanical excitation. The great interest is the harmonization of functional activity, in this case, electrodynamical and morphodynamical.

Due to the considerable complexity or sometimes the impossibility of such observations on full-scale objects, especially cell dimensions, modeling plays an invaluable role. Developed by the authors neural modeling technologies make it possible to organize the interaction of the elements of the modeled system as individually conditioned independently functioning fragments, and the degree of individuality can change indefinitely both in quantitative and qualitative senses.

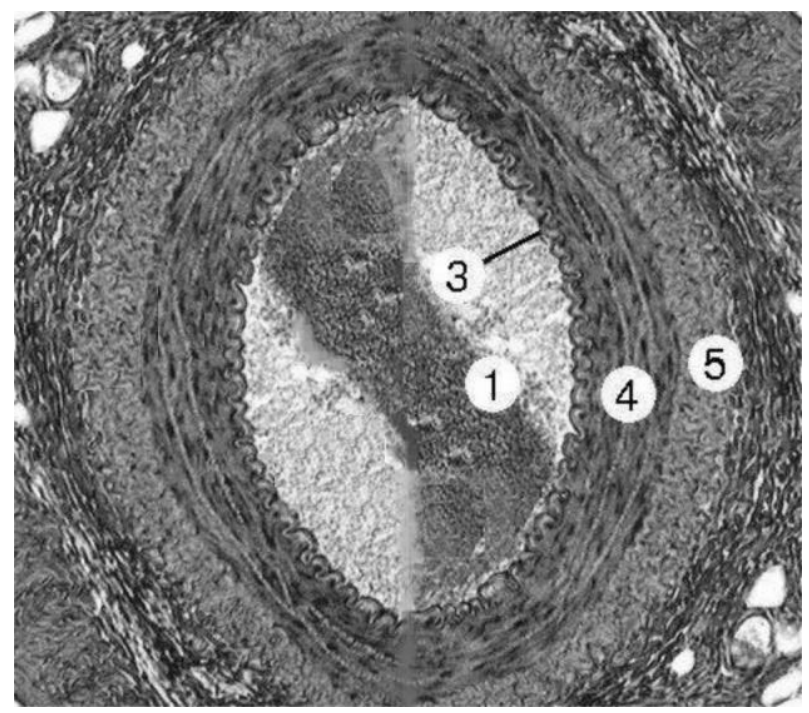

Fig.9. Artery Of Muscular Type Has A Lumen 1. In the Inner and the Middle Shells Visible as Dark Wavy Line - The Internal Elastic Membrane 3 With A Helical Internal Relief. Average Shell 4 is a Thick in an Artery and Consist of Helically Oriented Smooth Muscle Cells. 5 - Connective Fibrous Outer Shell. Coloring with Hematoxylin and Eosin.

The neurocomputer technologies allows organizing the interaction of the elements of the simulated system as individually conditioned independently functioning fragments, and the degree of individuality can change indefinitely both in quantitative and qualitative terms. The advantage of the proposed methodology of modeling and information technologies developed on its basis is the possibility of increasing the degree of individuality of elements both in quantitative and qualitative terms, expanding these differences hierarchically at different levels of complexity of any gradation. The limitation here can be only available computing power, which is surmountable using supercomputers or a distributed multi-user computing environment organized via the Internet.

Used neuron model reproduces the reflection in neuron electrical activity of morphodynamic processes of slow synaptic migration along the surface of dendrite processes that correlating with electrical synaptic activity. The reconciliation is carried out by a configurable finite-difference model of the dendrite, constructed on the operators of time shift by changing its wave characteristics in the intersynaptic sections under the effect of complex information processing of the input spike flow sequences over a set of parallel collectively interacting synaptic channels.

Changing the parameters and wave propagation time of electrical activity on the dendrite surface in the process of propagation simulates the intersynaptic collective communication not only in a functional, but also in a mutually-spatial ratio, that expressed in the dynamics of intersynaptic distances of the relative location of synapses on the dendrite. 


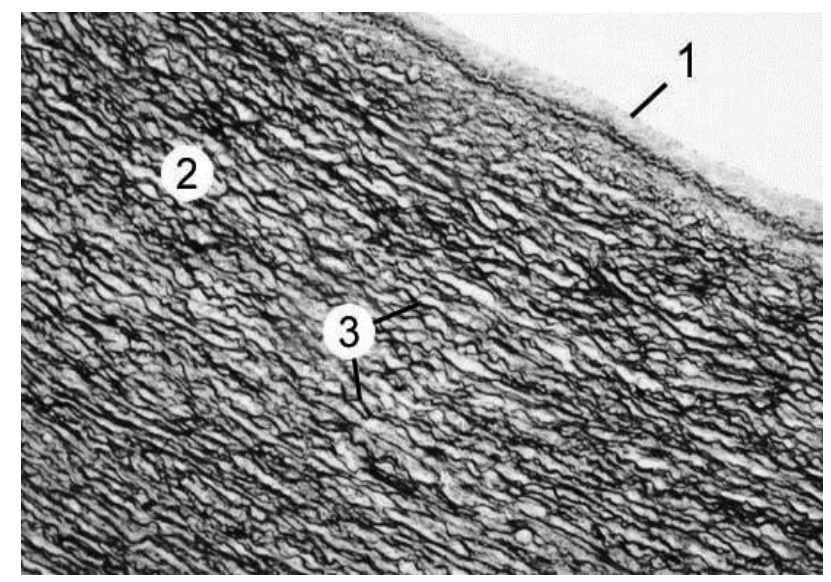

Fig.10. Aorta. The wall 1 consists of an Inner, Middle 2 and Outer Shells. Expressed Middle Shell Comprises Fenestrated Elastic Membranes 3.

At the same time, the inner endothelial sheath is thin (Fig. 9, tens of microns) and elastic, and therefore virtually follows the helix styling of powerful muscle fibers that control the lumen of the arteries, which is clearly seen in micrographs (Fig. 10).

As a result it was shown that the nature of blood flow significantly differs with the both quantitative and qualitative characteristics from the blood flow without regard to the spiral endothelial relief repeating helical laying of smooth shell. This effect is most expressed out to be cuts and relaxation of smooth muscles in dynamics.

\section{Conclusion}

The revision of conception of vascular coronary stent considering playback characteristics of the helical laying of smooth muscle membranes, with relief almost completely reproduced on the inner surface of a thin endothelial layer is required.

In this connection in case of not taking into consideration the analyzed factors, the straight walls are a source of disturbing of the natural flow of blood, which increases the likelihood of thrombus formation. Further, spiral flow, as is known [6,7], thereby reducing unicellular forms of parasitic populations, and disturbance such flow may increase reproductive ability of pathogenic microorganisms.

From a general theoretical point of view, the work allows the use of a common approach to the modeling of highly complex systems as a neurocomputing methodology and, more generally, a methodology for modeling the phenomenon of life.

Results may also be useful in the development of diagnostic and therapeutic simulator (modeling software and hardware), including using morphological 3D-technologies, their alignment with the functioning of the nervous tissue down to the cellular level - "revival" computer reconstructions morphology [29]. Such simulations can be applied for use in their actual morphometric data for diagnosis and therapy, as well as be used in training programs for students and post-graduate and continuing medical education.

The results of mathematical communicative-neurocomputer modeling applied to the cardiovascular system show that the double overlay method developed by the authors with the using of neural modeling techniques $[29,30]$ and bionics of spiral structures [31] can be effective in reproducing hidden effects that fundamentally determine the basic problem solving paradigm. This should contribute to the needs of developing a completely new direction in the implantable to cardiovascular system devices and new cardiac-gadgets design. 


\section{References}

[1] Folkow, B. \& Neil E. 1971. Circulation. Oxford University Press, New York, London, Toronto.

[2] T. J. Pedley 1980. The Fluid Mechanics of Large Blood Vessels (Cambridge Monographs on Mechanics).

[3] Bakusov LM Some of the models and methods of hemodynamic wave. Ufa: AIM. 1992. 50 pp.

[4] Leshchev SV Synergistic measurement neuro-platform: continual interfaces // Biomedical electronics. Number 2016. 4. pp.71-72.

[5] Kolushov VV, Saveliev AV Supervisualisation of biomehanodinamics of highly complex systems // Biomedical electronics. Number 4. 2015. pp 37-39.

[6] Yavelov IS, Stepanyan IV, Rochagov AV, Fedyanin VI, Zholobov AV (2017) Perspective Questions of Mechanopulsography. J Biomed Eng Med Devic 2: 125 DOI: 10.4172 / 2475-7586.1000125

[7] Yavelov IS, MY Izrailovich, Ehrlich BM Rochagov AV Modeling the effect of the massage effect on the increase in local blood flow. // Biomedical electronics. Number 2017. 3. S. 43-47.

[8] Nakayama Y, Furukoshi M, Terazawa T, Iwai R. Development of long in vivo tissue-engineered "Biotube" vascular grafts. Biomaterials. 2018; 185:232-239.

[9] Yang Y, Meyer CH, Epstein FH, Kramer CM, Salerno M. Whole-Heart Spiral Simultaneous Multi-Slice First-Pass Myocardial Perfusion Imaging. Magn Reson Med. 2018.

[10] Yahyaie M, Tarokh MJ, Mahmoodyar MA. Use of Internet of Things to Provide a New Model for Remote Heart Attack Prediction. Telemed J E Health. 2018.

[11] Yıldırım Ö, Pławiak P, Tan RS, Acharya UR. Arrhythmia detection using deep convolutional neural network with long duration ECG signals. Comput Biol Med. 2018.

[12] Meyer A, Zverinski D, Pfahringer B, et al. Machine learning for real-time prediction of complications in critical care: a retrospective study. Lancet Respir Med. 2018.

[13] He R, Wang K, Zhao N, et al. Automatic Detection of Atrial Fibrillation Based on Continuous Wavelet Transform and 2D Convolutional Neural Networks. Front Physiol. 2018; 9:1206.

[14] Iffrig E, Wilson JS, Zhong X, Oshinski JN. Demonstration of circumferential heterogeneity in displacement and strain in the abdominal aortic wall by spiral cine DENSE MRI. J Magn Reson Imaging. 2018.

[15] Sander B. MR-Grundlagen E-print: http://www.mrx.de/mrpraxis/mrgrund.html

[16] White DN The early development of neurosonology: I. echoencephalography in adults // Ultrasound in Medicine and Biology. 1992. V.18. \# 2. P.115-165.

[17] Saveliev A.V. The law of conservation of complexity and its application in problems of modeling nonequilibrium systems. // Simulation of non-equilibrium systems. Krasnoyarsk. 1998. p. 100-101.

[18] Saveliev A.V. Towards a general theory of neural networks. On the issue of complexity // Neurocomputers: development and application. Radio engineering, Moscow. 2006. № 4-5. Pp. 4-14.

[19] Kolushov VV, Saveliev AV The methodology of individual collective modeling neural biovozbudimosti as a new paradigm neurocomputing // Neurocomputers: development and application. 2010. 8. S.25-34 number, http://www.ec-ai.ru/default.asp?article=3.

[20] Kolushov VV Application of neural network algorithms in the realization of expert modeling technology cardiodynamic // In: Neuroinformatics-2004: Proceedings of the VI All-Russia. NTK. M.: MEPHI, 2004. V.2.

[21] Kositsin NSThe microstructure of dendrites and aksodendriticheskih connections in the central nervous system. M.: Nauka. 1976. 199 p.

[22] Shotton DM, Heuser JE, Reese BF, Reese TS Postsynaptic membrane folds of the frog neuromuscular junction visualized by scanning electron microscopy // Neuroscience. 1979. V.4. \# 3, P.427-435. 
[23] Kerlov IV Art of 3D-animation and special effects. (Per.s Engl. Smolina EV). M.: OOO "Top", 2004. $480 \mathrm{p}$.

[24] Ratner P. Three-dimensional modeling and animation of man. M: Williams. 2005. $272 \mathrm{p}$.

[25] Saveliev A.V. Neurological aspects of cellular neuromathematics // Artificial Intelligence. NAS of Ukraine, Donetsk. 2008. No. 4. P. 612-623.

[26] Saveliev A.V. Neurophysics of the brain and neuro-modeling: diffusion-synergistic aspects of volumetric neurotransmitting // Journal of Open Systems Evolution. Kazakhstan, Almaty. 2008. No. 1. V. 9 P. 93-101.

[27] Patent DE 618610 C. Wasserfuehrung / Schauberger V. 1935-09-12.

[28] Patent AT196680 B. Rohrleitung für flüssige und gasförmige Medien / Schauberger V. 1958-03-25.

[29] Bryantsev IS, Kolushov VV, Saveliev AV "Virtual revival" of neural information objects // Neurocomputers: development and application. 2016. №5. pp. 21-23.

[30] Mishustin IA, Preobrazhensky NB, Zhdanov A.A., Stepanyan I.V. Hardware implementation of a neuron-like network with the ability to recognize noisy images // Neurocomputers: development, application. 2018. № 6. S. 19-25.

[31] Petoukhov S.V., Svirin V.I., Khazina L.V. Bionics of Spiral Structures. - Journal of Machinery Manufacture and Reliability, 2015, Vol. 44, No. 3, pp. 249-253. (C) Allerton Press, Inc., 2015.

\section{Authors' Profiles}

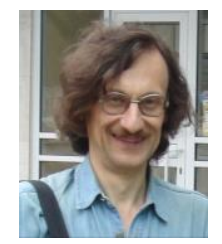

Alexander Savelyev - p.h.d. chief editor of the magazine "Neurocomputers: development, application" of "Radio Engineering" Publisher, scientific coordinator of "Neurophilisophy" seminar, Faculty of Philosophy of Moscow State University named after M.V. Lomonosov, secretary of the seminar "Knowledge Management" of the Government administration department of the Moscow State University (Moscow), a member of the organizing committee of the international interdisciplinary congress "Neuroscience for Medicine and psychology" (the Crimea, Sudak). Ph.D. thesis (2016) "Philosophical and methodological foundations of neurocomputing" is specializing in the philosophy of science and technology at the Faculty of Philosophy of Moscow State University named after M.V. Lomonosov. Scientific interests: biomechanics and medical technology, neurocybernetics, neurobiology, measuring equipment, anthropology (was invited to the post of editor of the journal Current Anthropology of the University of Chicago), valeology, modeling of the nervous system, ways and devices to enhance the capacity of the brain; geophysics; search works and equipment.

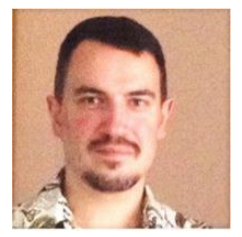

Ivan Stepanyan - Doctor of Biologicals sciences (Sc.D.), Ph.D., leading research scientist, Laboratory of biomechanic systems of Institute of Machine Science named after A.A. Blagonravov of the Russian Academy of Sciences, Research Institute of Occupational Health named after N.F. Izmerov of the Russian Academy of Sciences, Moscow State Conservatory named after P.I. Chaikovsky. Author of more than 140 scientific works. Graduated National University of Science and Technology (MSMU, 2003). Theme of the master's dissertation (2003) is "Information processes modeling with extended Petri nets", where investment flows in regions of Russian Federation were mathematically modeled. Theme of candidate dissertation (2006) is "Neural network algorithms of recognizing the results of acoustic spirometry". Theme of doctoral dissertation (2012) "Scientific and methodological foundations and bioinformation technologies of risk management in occupational health". Co-founder of Pentagramon. Scientific interests: algebraic biology, matrix genetics, biomechanics, bioinformatics, medical devices, phyllotaxis, science-art, disappearing languages, occupational medicine, quantum gravitation, programming theory, artificial intelligence. 
How to cite this paper: A.V. Savelyev, I.V. Stepanyan,"Spiral Flows at the Cardiovascular System as the Experimental Base of New Cardiac-gadgets Design", International Journal of Engineering and Manufacturing(IJEM), Vol.8, No.6, pp.1-12, 2018.DOI: 10.5815/ijem.2018.06.01 